\title{
A Computer Program for Dynamic Load Simulation of Spur Gears with Asymmetric and Symmetric Teeth
}

\author{
Fatih Karpat ${ }^{1}$, Stephen Ekwaro-Osire ${ }^{2}$, Esin Karpat $^{3}$ \\ ${ }^{1}$ Department of Mechanical Engineering, Uludag University, Bursa, Turkey \\ ${ }^{2}$ Department of Mechanical Engineering, Texas Tech University, Lubbock, USA \\ ${ }^{3}$ Department of Electronics Engineering, Uludag University, Bursa, Turkey \\ Email: karpat@uludag.edu.tr, stephen.ekwaro-osire@ttu.edu, esinoz@uludag.edu.tr
}

Received August 2, 2012; revised September 1, 2012; accepted September 12, 2012

\begin{abstract}
An important concern in gear design is to reduce the dynamic load and noise of gear systems. It has been found that the noise generated from gearing is basically due to gearbox vibration excited by the dynamic load. Since one of the situations that demand high performance is the high rotational speeds, there is a need to understand the dynamic behavior of the gears at such speeds. Such knowledge would shed light on detrimental characteristics like dynamic loads and vibrations. An efficient way in performing studies on the dynamic behavior of gears is using computer aided analysis on numerical models. In this paper, a developed computer program is introduced to analyze dynamic behavior of spur gears with asymmetric teeth that have a potential use for higher performance in wind turbine gearboxes. This program can be used to compare conventional spur gears with symmetric teeth and spur gears with asymmetric teeth. By using this program, gear designers can design a gear pair and obtain results, e.g. dynamic Load, transmitted torque, static transmission error, and frequency spectra of static transmission error etc., just by pressing a command button.
\end{abstract}

Keywords: Gears; Computer Program; Asymmetric Teeth

\section{Introduction}

Gear dynamics has been a subject of intense interest to the gearing area during the last few decades dynamic loads and vibration are a major concern for gear drives at high speeds. Simulation of meshing of gear drives performed by application of tooth contact analysis and experimental tests of gear drives have confirmed that transmission errors (TE) are the prime cause of noise and vibrations of the gear drives [1]. The definition of transmission error is made as "The difference between the actual position of the output gear and the position it would occupy if the gear drive were perfectly conjugate". This may be expressed as angular displacement or as linear displacement at the pitch point [2]. The causes of transmission error are elastic deflections under load, geometrical errors and geometrical modifications.

Transmission errors causing dynamic loads in gears affect not only the gear vibrations and noise but also tooth fatigue, and surface failure. Therefore, the most important objective in gear design is the minimization of dynamic loads and transmission errors.

There have been many studies on gear design in literature. The prediction of gear transmission errors and gear dynamic loads, gear noise and vibration for gear drives are always main concerns in gear design. Com- prehensive reviews on the development of a variety of simulation models for both static and dynamic analysis of different types of gears are presented in [3-5]. Tearuchi and Hidetaro [5] used the tooth deflection, equivalent composite error, and equivalent mass of gear, in the calculation of the dynamic loads on gear teeth. The numerical results obtained were shown to be in good agreement with the experimental results. A similar vibratory model was presented in [6]. A comparison of the theoretical and the experimental results, obtained for dynamic characteristics of the heavily loaded spur gears, was made. A numerical approach for the equations of motion that contain the excitation terms due to errors and periodic variation of the mesh stiffness was developed and presented. This method was adapted and employed by several researchers [7-14] to calculate the dynamic contact load or the torsional response, depending on different gear parameters, i.e. tooth errors, addendum modification, mesh stiffness, lubrication, damping factor, gear contact factor, and friction coefficient. In gear design, the dynamic factor is generally used to quantify the dynamic effects. In this context, the dynamic factor is defined as the ratio of the maximum dynamic load to the maximum static load on the gear tooth. Dynamic loads of gears with low contact ratio (contact ratio is between 1 and 2) are affected by several parameters, namely, time-varying 
mesh stiffness, tooth profile error, contact ratio, friction, and sliding. The static transmission errors change in a periodic manner due to the variation of gear mesh stiffness during contact. This is the source of vibratory excitation in gear dynamics. The static transmission error has basic periodicities related to the shaft rotational frequencies and the gear mesh frequency. The mesh frequency and its first harmonics are the predominant contributors to the generation of noise. Many researchers investigated the effects of different parameters (e.g. design load and tooth profile modification) in decreasing the static transmission errors [11-13]. In addition, the Fast Fourier Transform (FFT) can be used to perform the frequency analysis of static transmission error.

Since the variation of gear-pair meshing tooth stiffness causes static transmission errors, one of the most important method to minimize transmission errors is to change the gear tooth stiffness. In involute gears, high contact gear ratio gears and non standard gears are used to vary the gear tooth stiffness. Recently, involute spur gears with asymmetric teeth provide flexibility to designers for different application areas due to non-standard design. If they are correctly designed, they can make important contributions to the improvement of designs in aerospace industry, automobile industry, and wind turbine industry. This often relates to improving the performance, increasing the load capacity, reduction of acoustic emission, and reduction of vibration.

A number of studies on the design and stress analysis of asymmetric gears are available in literature. A large number of studies have been performed over the last two decades to assess whether asymmetric gears are an alternative to conventional gears in applications requiring high performance. In these studies, some standards (e.g. ISO 6336 and DIN 3990), analytical methods (e.g. the Direct Gear Design method and the tooth contact analysis), and numerical methods (Finite elements methodFEM) have been used to compare the performance of conventional and asymmetric gears under same conditions [15-18].

In order to utilize asymmetric gear designs more effecttively, it is imperative to perform analyses of these gears under dynamic loading. In some studies [19-22], preliminary results related on the response of asymmetric gears under dynamic loading are presesented. The effect of some design parameters, such as pressure angle or tooth height on dynamic loads, was shown. Although asymmetric tooth is emerging as a major concern in gear researches, in literature, by now there was not any virtual tool for design of spur gears with asymmetric teeth. Therefore, in this study, a MATLAB-based virtual tool called DYNAMIC to analyze dynamic behavior of spur gears with asymmetric teeth depending on various tooth parameters. The objective of this paper is to introduce a developed MATLAB-based virtual tool called DYNAMIC and to demonstrate its capabilities.

\section{Dynamic Analysis of Gears}

\subsection{Dynamic Model}

During one mesh period the tooth contact load in one gear pair does not stay constant. This load varies depending on the transition from double tooth contact to single tooth contact.

To determine the variation of dynamic load as a function of the contact position (or time), it is necessary to derive the equations of motion for gear tooth pair in a mesh. Considering the free body diagrams of the gear and pinion shown in Figure 1, the equations of motion can be formulated as:

$$
\begin{aligned}
& J_{g} \ddot{\theta}_{g}=r_{b g}\left(F_{I}+F_{I I}\right) \pm \rho_{g I} \mu_{I} F_{I} \pm \rho_{g I I} \mu_{I I} F_{I I}-r_{b g} F_{D} \\
& J_{p} \ddot{\theta}_{p}=r_{b p} F_{D}-r_{b p}\left(F_{I}+F_{I I}\right) \pm \rho_{p I} \mu_{I} F_{I} \pm \rho_{p I I} \mu_{I I} F_{I I}
\end{aligned}
$$

where $J_{p}$ and $J_{g}$ represent the polar mass moments of inertia of the pinion and gear, respectively. The dynamic contact loads are $F_{I}$ and $F_{I I}$, while $\mu_{I}$ and $\mu_{I I}$ are the instantaneous coefficients of friction at the contact points. $\theta_{p}$ and $\theta_{g}$ represent the angular displacements of pinion and gear. The radii of the base circles of the engaged gear pair are $r_{b p}$ and $r_{b g}$, while the radii of curvature at the mating points are $\rho_{p I, I I}$ and $\rho_{g l, I I}$.

In above equations, if the speed of the pinion tooth is greater than the speed of the gear tooth, the sign of the friction force is positive, otherwise it is negative. The static tooth load is defined as:

$$
F_{D}=\frac{T_{p}}{r_{b p}}=\frac{T_{g}}{r_{b g}}
$$

If the angular coordinate is converted into the coordinate along the line of action, the displacements of the

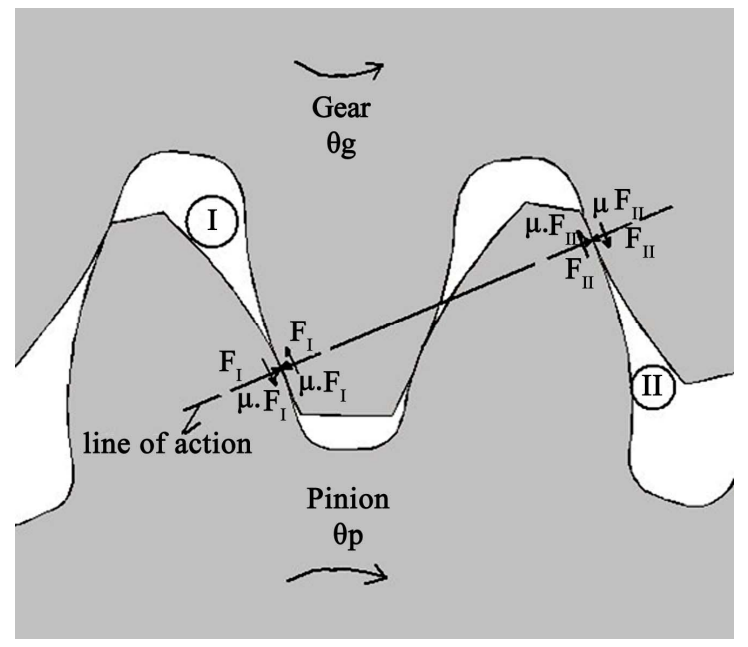

Figure 1. Engaging teeth pairs along the line of action. 
undeformed tooth profiles, along the line action, can be written as:

$$
\begin{aligned}
& y_{g}=r_{g} \cdot \theta_{g} \\
& y_{p}=r_{p} \cdot \theta_{p}
\end{aligned}
$$

The relative displacement, velocity, and acceleration can then be cast as:

$$
\begin{gathered}
x_{r}=y_{p}-y_{g} \\
x_{s}=\frac{\left(M_{g}+M_{p}\right.}{} \\
M_{p}=J_{p} / r_{b p}^{2} \\
M_{g}=J_{g} / r_{b g}^{2}
\end{gathered}
$$$$
X_{S}=\frac{\left(M_{g}+M_{p}\right) F_{D}+K_{I} \cdot \lambda_{I}\left(S_{p I} \cdot M_{g}+S_{g I} \cdot M_{p}\right)+K_{I I} \cdot \lambda_{I I}\left(S_{p I I} \cdot M_{g}+S_{g I I} \cdot M_{p}\right)}{K_{I} \cdot\left(S_{p I} \cdot M_{g}+S_{g I} \cdot M_{p}\right)+K_{I I} \cdot\left(S_{p I I} \cdot M_{g}+S_{g I I} \cdot M_{p}\right)}
$$

The effective gear masses are:

termination of errors.

$$
\begin{aligned}
& \dot{x}_{r}=\dot{y}_{p}-\dot{y}_{g} \\
& \ddot{x}_{r}=\ddot{y}_{p}-\ddot{y}_{g}
\end{aligned}
$$

Including viscous damping, the equations of motion reduce to:

$$
\ddot{x}_{r}+2 \omega \xi \dot{x}_{r}+\omega^{2} x_{r}=\omega^{2} x_{s}
$$

The loaded static transmission errors can be obtained:
The equivalent stiffness of meshing tooth pairs can be written as:

$$
\begin{gathered}
K_{I}=\frac{k_{p I} \cdot k_{g I}}{k_{p I}+k_{g I}} \\
K_{I I}=\frac{k_{p I I} \cdot k_{g I I}}{k_{p I I}+k_{g I I}}
\end{gathered}
$$

The friction experienced by the pinion and the gear can be expressed as:

$$
\begin{gathered}
S_{p I}=1 \pm \frac{\mu_{I} \cdot \rho_{p I}}{r_{b p}} \\
S_{g I}=1 \pm \frac{\mu_{I} \cdot \rho_{g I}}{r_{b d}} \\
S_{p I I}=1 \pm \frac{\mu_{I I} \cdot \rho_{p I I}}{r_{b p}} \\
S_{g I I}=1 \pm \frac{\mu_{I I} \cdot \rho_{g I I}}{r_{b d}}
\end{gathered}
$$

The signs in the above expressions are positive $(+)$ for the approach and negative $(-)$ for the recess. The dynamic contact loads, which include tooth profile error, can then be written as:

$$
\begin{aligned}
& F_{I}=K_{I}\left(x_{r}-\lambda_{I}\right) \\
& F_{I I}=K_{I I}\left(x_{r}-\lambda_{I I}\right)
\end{aligned}
$$

where $\lambda_{I}$ and $\lambda_{I I}$ are the tooth profile errors. In this paper, the effects of profile errors on the dynamic response of gears are not considered. Thus, the tooth profile errors are assumed to be zero. The developed computer program has a capability of using any approach for the de-
The reduced equation of motion in Equation (1) and (2) is solved numerically using a method previously detailed in Reference [19-20]. This method employs a linearized iterative procedure that involves dividing the mesh period into many equal intervals. In this study, a MATLAB program is developed. The flowchart of this computational procedure used for calculating the dynamic responses of spur gears, is shown in Figure 2. The time interval, between initial contact point and the highest point of single contact is considered as a mesh period. In the numerical solution, each mesh period is divided into 200 points for good accuracy. Within a small interval, various parameters of equations of motion are taken as constants and an analytical solution obtained. The calculated values of the relative displacement and the relative velocity after one mesh period are compared with the initial values $x_{r}$ and $v_{r}$. Unless the differences between procedure is repeated by taking the previous calculated

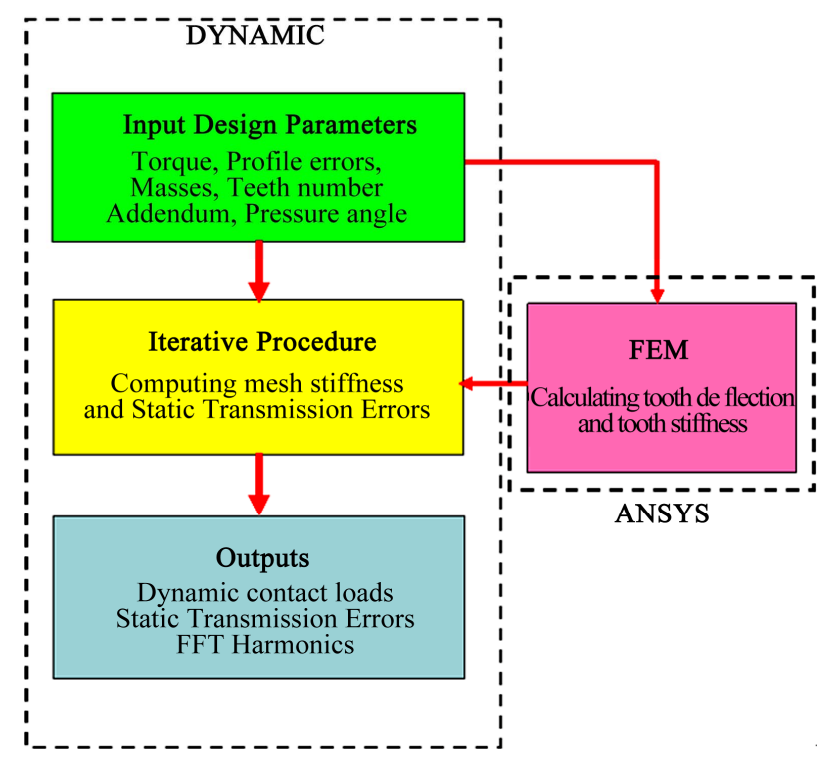

Figure 2. The structure of developed computer program. 
them are smaller than a preset tolerance, the iteration $x_{r}$ and $v_{r}$ at the end point of single pair teeth contact as new initial conditions. Then the dynamic loads are calculated by using the calculated relative displacement values.

After the gear dynamic load has been calculated, the dynamic load factor can be determined by dividing the maximum dynamic load along contact line to the static load. The dynamic factor indicates the instantaneous increase of gear tooth load over the static load. It is one of the most important parameters used for understanding the dynamic responses of gear drives.

In literature, different methods and empirical equations are used to calculate the tooth deflections of spur gears. These methods are often based on the classical theory of elasticity and numerical approaches. However, all of them are derived for symmetric tooth. Therefore, in this study, a 2-D finite element model is developed to calculate the deflections of both the asymmetric and the symmetric gear teeth (Figure 3). A computer program, which saves time and provides a means to carry out a parametric study with the gear parameters, was developed using MATLAB. This program generates batch files for input into ANSYS. When this file is executed in ANSYS, the general procedure of FEA (i.e. 2D modeling, meshing, loading, solution, and post processing) is automatically performed. At the end, an output file, that contains nodal deflection for loaded nodes, is created. This process is

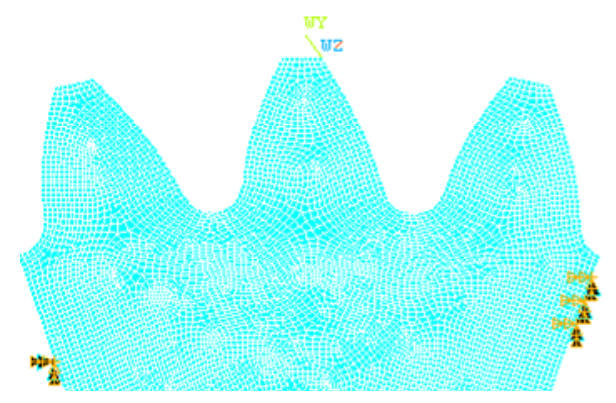

Figure 3. 2-D finite element model.

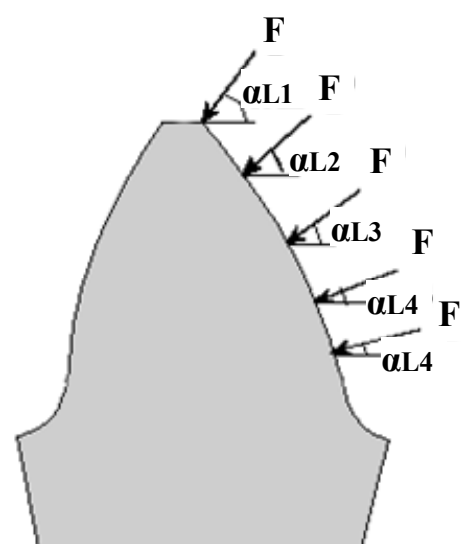

Figure 4. Load application. repeated for each gear. It should be noted that in this analysis the loads are applied at five locations on the gear files, the approximate curves for the single tooth stiffness, along the contact line, are obtained with respect to the radius of the gears.

To facilitate the calculation of the Hertzian component of the deflection at the point loading, the size of the grid near the point of loading is chosen as recommended by $[19,21]$ using the following equation:

$$
\frac{\mathrm{e}}{b_{h}}=-0.2\left(\frac{c}{e}\right)+1.2 \text { for } 0.9 \leq \frac{c}{\mathrm{e}} \leq 3
$$

where $c$ and $e$ are the length and width of the element, respectively. And $b_{h}$ is the Hertzian contact width:

$$
b_{h}=2.15 \sqrt{\frac{F \cdot\left(2 \cdot \rho_{p} \cdot \rho_{d} /\left(\rho_{p}+\rho_{d}\right)\right)}{E}}
$$

tooth (Figure 4). 8-noded parabolic isoparametric elements are used for meshing of the 2D model. By using the nodal deflection values that are read from the output where $F$ is applied load per unit length and $E$ is Young's modulus of gear material.

\subsection{Virtual Tool: DYNAMIC}

Physics-based modeling and simulation is important in all engineering problems. The current mature stage of computer software and hardware makes it possible for complex mechanical problems, such as gear design, to be solved numerically. In-house prepared codes to handle individual research projects, graduate, and/or $\mathrm{PhD}$ studies; commercial packages for engineers in industry are widely used to solve almost every engineering problem. Tailored with graphical user interfaces (GUIs) and easyto-use design steps, anyone-even a beginner can design a gear pair and obtain results, e.g. Dynamic Load, Transmitted Torque, Static Transmission Error as a function of time, and Static Transmission Error Harmonics etc., just by pressing a command button. Lecturers have been increasingly using these packages to increase their teaching performance and student understanding.

Based on and triggered by these thoughts, a virtual tool DYNAMIC is prepared that can be used for educational and research purposes. The DYNAMIC is a general purpose tool for gear analysis (Figure 5).

There are six blocks and a figure block on the front panel of the tool. Three blocks on the right side of the front panel, belong to the parameters which will be defined by the users. Pinion and Gear blocks are reserved for the tooth parameters and Mechanism block is for the parameters related to the mechanical variables. The two blocks above the figure are Simulation and Figure Selection panels. Once the user inputs the needed parameters, he/she clicks the CALCULATE pushbutton to obtain the 
solution for the specified parameters. In the Figure Selection block, from the pop-up menu, user can select which solution to be plotted: Dynamic Load, Transmitted Torque, Static Transmission Error or Static Transmission Error Harmonics. Then the required figure can be plotted with the PLOT button. Once the solutions are calculated, it is not needed to run the program again and again for each figure option. CLEAR is to clean the figure axes before each plot.

The variation of dynamic load, static transmission errors, static transmission error harmonics and, transmitted torque with respect to time can be seen in Figures 6(a)(c). The solutions for different variables can be plotted in one figure, for comparison. Figure 6(a) shows a sample result for the variation of the static transmission error during a mesh period. In Figure 6(b), the frequency spectra of the static transmission errors obtained by using the fast Fourier transform (FFT) are depicted. DYNAMIC program can also provide transmitted torque results respect to time for sample gear pairs.

\section{Conclusion}

In this paper, a MATLAB-based virtual tool, DYNAMIC, is introduced to analyze dynamic behavior of spur gears with asymmetric tooth design. The DYNAMIC can be used to compare conventional spur gears with symmetric teeth and spur gears with asymmetric teeth. The results for dynamic load, dynamic factor, transmitted torque, static transmission error and static transmission error harmonics can be obtained for various tooth parameters to show the powerful aspects of asymmetric teeth. Influence of various parameters (e.g. the pressure angle on drive side or coast side, addendum, and teeth number) on the static transmission errors, the amplitudes of harmonics of the static transmission errors and the dynamic loads can be investigated. By using this program, gear designers can design a gear pair and obtain results, e.g. dynamic load, transmitted torque, static transmission error, and frequency spectra of static transmission error etc., just by pressing a command button.

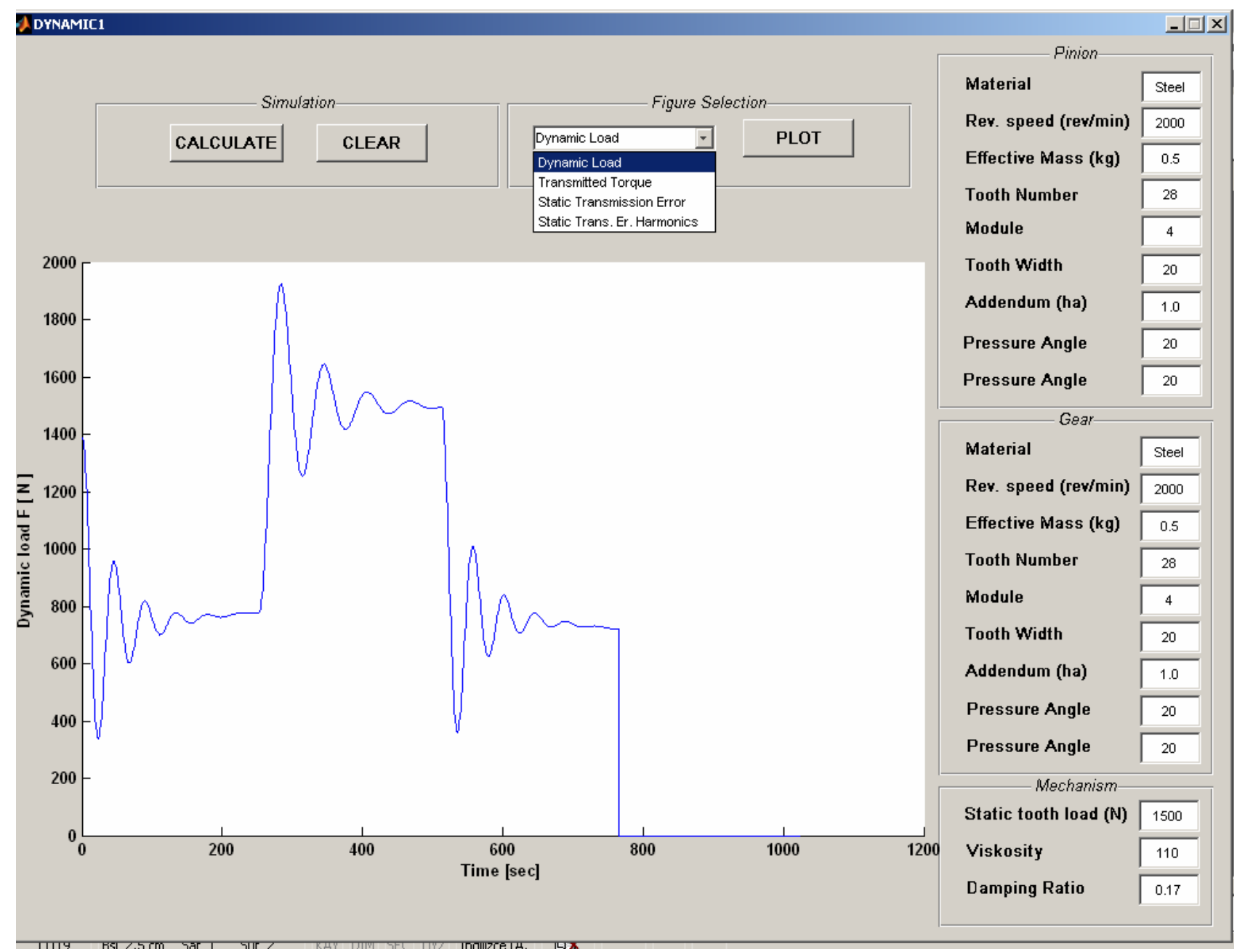

Figure 5. The front panel of the DYNAMIC tool. 


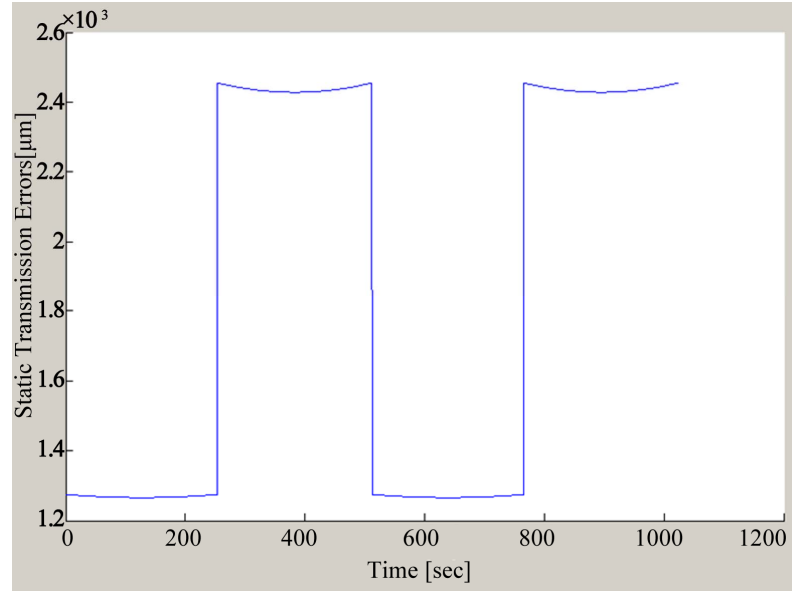

(a)

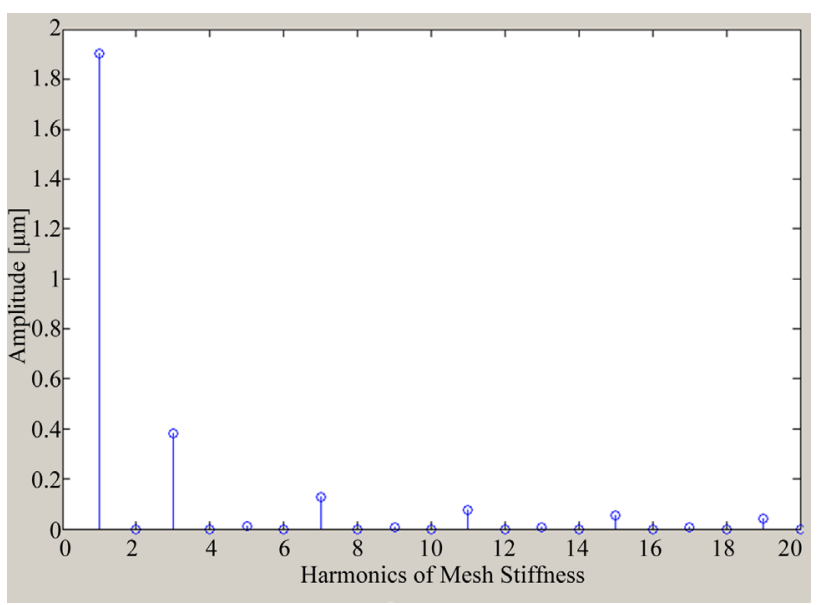

(b)

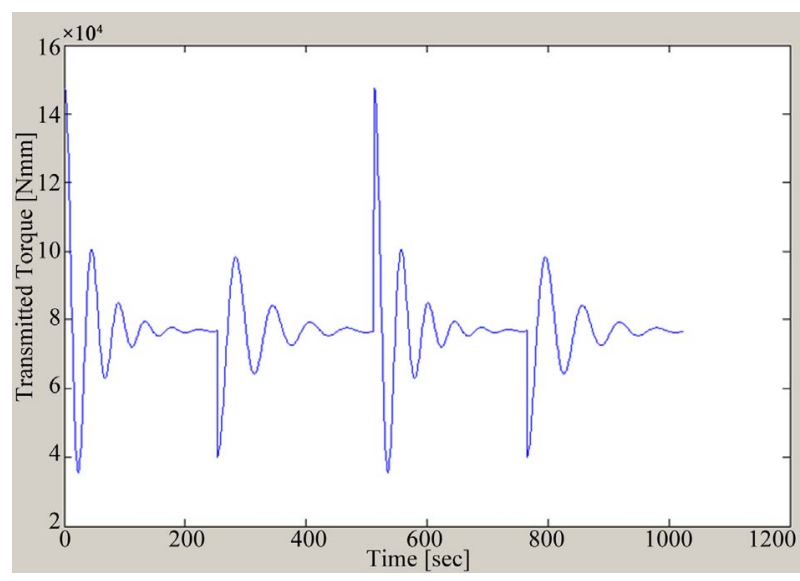

(c)

Figure 6. The variation of static transmission errors (a), static transmission error harmonics (b) and, transmitted torque (c) with respect to time.

\section{Acknowledgements}

The authors gratefully acknowledge the support of Uludag University under grant BAP-YDP(M) 2010/10 and
Texas Tech University under grant DE-FG36-06-GO 86092 .

\section{REFERENCES}

[1] F. L. Litvin, D. Vecchiato, K. Yukishima, A. Fuentes, I. Gonzalez-Perez and K. Hayasaka, "Reduction of Noise of Loaded and Unloaded Misaligned Gear Drives," Computer Methods in Applied Mechanics and Engineering, Vol. 195, No. 41-43, 2006, pp. 5523-5536. doi:10.1016/j.cma.2005.05.055

[2] M. Åkerblom, "Gear Noise and Vibration-A Literature Survey," Report, Maskinkonstruktion, Stckholm, 2001, p. 26. http://urn.kb.se/resolve?urn=urn:nbn:se:kth:diva-9891

[3] H. N. Ozguven and D. R. Houser, "The Mathematical Models Used in Gear Dynamics-A Review," Journal of Sound and Vibration, Vol. 121, No. 3, 1988, pp. 383-411. doi:10.1016/S0022-460X(88)80365-1

[4] A. Parey and N. Tandon, "Spur Gear Dynamic Models Including Defects: A Review," Shock Vibration Digest, Vol. 35, No. 6, 2003, pp. 465-78. doi: $10.1177 / 05831024030356002$

[5] Y. Tearuchi and M. Hidetaro, "Comparison of Theories and Experimental Results for Surface Temperature of Spur Gear Teeth," Journal of Engineering for Industry, Transactions of the ASME, Vol. 96, No. 11, 1974, pp. 41-50.

[6] K. Ichimaru and F. Hirano, "Dynamic Behavior of Heavy-Loaded Spur Gears," Journal of Engineering for Industry, Transactions of the ASME, Vol. 96, No. 2, 1974, pp. 373-381.

[7] K. L. Wang and H. S. Cheng, "Numerical Solution to the Dynamic Load, Film Thickness, and Surface Temperatures in Spur Gears - Part 2 Results," Journal of Mechanical Design, Vol. 103, No. 4, 1981, pp. 188-194. doi:10.1115/1.3254860

[8] K. L. Wang, H. S. Cheng, K. L. Wang and H. S. Cheng, "Numerical Solution to the Dynamic Load, Film Thickness, and Surface Temperatures in Spur Gears-Part 1. Analysis," Journal of Mechanical Design, Vol. 103, No. 4, 1981, pp. 177-187. doi:10.1115/1.3254859

[9] S. M. A. Arikan, "Dynamic Load and Contact Stress Analysis of Spur Gears," 17th Design Automation Conference Presented at the 1991 ASME Design Technical Conferences, Miami, 22-25 September 1991, pp. 85-91.

[10] J. H. Kuang and A. D. Lin, "The Effect of Tooth Wear on the Vibration Spectrum of a Spur Gear Pair," Journal of Vibration and Acoustics-Transactions of the Asme, Vol. 123, No. 3, 2001, pp. 311-317. doi:10.1115/1.1379371

[11] J. H. Kuang, A. D. Lin, J. H. Kuang and A. D. Lin, "Theoretical Aspects of Torque Responses in Spur Gearing Due to Mesh Stiffness Variation," Mechanical Systems and Signal Processing, Vol. 17, No. 2, 2003, pp. 255-271. doi:10.1006/mssp.2002.1516

[12] A. Andersson, "An Analytical Study of the Effect of the Contact Ratio on Spur Gear Dynamic Response," Journal of Mechanical Design, Vol. 122, No. 4, 2000, pp. 508514. doi:10.1115/1.1320819 
[13] R. Kasuba and J. W. Evans, "An Extended Model for Determining Dynamic Loads in Spur Gearing," Journal of Mechanical Design, Vol. 103, No. 2, 1981, pp. 398409. doi:10.1115/1.3254920

[14] A. S. Kumar, T. S. Sankar and M. O. M. Osman, "On Dynamic Tooth Load and Stability a Spur-Gear System Using the State-Space Approach," Journal of Mechanisms, Transmissions, and Automation in Design, Vol. 107, No. 1, 1985, pp. 54-60. doi:10.1115/1.3258695

[15] K. Cavdar, F. Karpat and F. C. Babalik, "Computer Aided Analysis of Bending Strength of Involute Spur Gears with Asymmetric Profile," Journal of Mechanical Design, Vol. 127, No. 3, 2005, pp. 477-484.

[16] A. Kapelevich, "Geometry and Design of Involute Spur Gears with Asymmetric Teeth," Mechanism and Machine Theory, Vol. 35, No. 1, 2000, pp. 117-130. doi:10.1016/S0094-114X(99)00002-6

[17] F. Karpat and S. Ekwaro-Osire, "Influence of Tip Relief Modification on the Wear of Spur Gears with Asymmetric Teeth," Tribology Transactions, Vol. 51, No. 5, 2008, pp. 581-588. doi:10.1080/10402000802011703

[18] F. Karpat, S. Ekwaro-Osire and M. P. H. Khandaker,
"Probabilistic Analysis of MEMS Asymmetric Gear Tooth," Journal of Mechanical Design, Vol. 130, No. 4, 2008, pp. 042306.1-042306.6.

[19] F. Karpat and S. Ekwaro-Osire, "Influence of Tip Relief Modification on the Wear of Spur Gears with Asymmetric Teeth," Tribology \& Lubrication Technology, Vol. 66, No. 6, 2010, pp. 50-60.

[20] F. Karpat, S.Ekwaro-Osire, K.Cavdar and F.C. Babalik, "Dynamic Analysis of Involute Spur Gears with Asymmetric Teeth," International Journal of Mechanical Sciences, Vol. 50, No. 12, 2008, pp. 1598-1610. doi:10.1016/j.ijmecsci.2008.10.004

[21] J. J. Coy and C. H. C. Chao, "Method of Selecting Grid Size to Account for Hertz Deformation in Finite Element Analysis of Spur Gears," Journal of Mechanical Design, Vol. 104, No. 4, 1982, pp. 759-766. doi:10.1115/1.3256429

[22] R. Muthukumar and M. R. Raghavan, "Estimation of Gear Tooth Deflection by the Finite Element Method," Mechanism and Machine Theory, Vol. 22, No. 2, 1987, pp. 177-181. doi:10.1016/0094-114X(87)90042-5 